\title{
Phase shift in atom interferometers: Corrections for nonquadratic potentials and finite-duration laser pulses
}

\author{
A. Bertoldi \\ LP2N, Laboratoire Photonique, Numérique et Nanosciences, Université Bordeaux-IOGS-CNRS:UMR 5298, F-33400 Talence, France \\ F. Minardi* \\ Istituto Nazionale di Ottica, CNR-INO, 50019 Sesto Fiorentino, Italy; \\ LENS and Dipartimento di Fisica e Astronomia, Università di Firenze, 50019 Sesto Fiorentino, Italy; \\ and Dipartimento di Fisica e Astronomia, Università di Bologna, 40127 Bologna, Italy \\ M. Prevedelli \\ Dipartimento di Fisica e Astronomia, Università di Bologna, 40127 Bologna, Italy
}

(Received 31 December 2018; published 25 March 2019)

\begin{abstract}
We derive an expression for the phase shift of an atom interferometer in a gravitational field taking into account both the finite duration of the light pulses and the effect of a small perturbing potential added to a stronger uniform gravitational field, extending the well-known results for rectangular pulses and at most quadratic potentials. These refinements are necessary for a correct analysis of present-day high-resolution interferometers.
\end{abstract}

DOI: 10.1103/PhysRevA.99.033619

\section{INTRODUCTION}

Atom interferometry rests upon the coherent manipulation of matter waves [1]. The increasing ability to control individual quantum systems and their evolution makes it feasible to observe quantum interference over trajectories with very large separation in momentum [2,3] and space [4]. The resulting high sensitivity and the exquisite control of systematic effects are at the basis of the growing number of applications in atom interferometry, ranging from tests of general relativity $[5,6]$, the measurement of fundamental constants $[7,8]$, and the search for new physics $[9,10]$ to more applied contexts like inertial navigation [11].

The improving experimental performances of atom interferometry require a refinement of the modeling for the phase shift calculation. Two main formulations have been developed to obtain the interferometric phase shift in the case of two-path configurations, with three or more light pulses: a path integral approach [12-16] and a density matrix equation in the Wigner representation [17]. Several effects have been investigated especially in the first formulation, such as the finite speed of light [18] or the wave-front aberration of the light beams [19]. The calculation has been also extended to the general relativistic case [20,21].

We adopt here a formalism based on the Heisenberg representation to describe the dynamics of a two-level atom in an external potential coherently manipulated with a pulsed laser beam [22]; this formulation provides the interferometric phase by adopting a series of unitary transformations to write the evolution operator in simple terms. First, we calculate the dependence of the interferometric phase on the finite

\footnotetext{
*francesco.minardi@ino.cnr.it
}

pulse duration, previously treated in Refs. [23-25]: the result in Eq. (21) agrees with the existing literature and is valid for pulses of arbitrary shape. Our approach can be extended to calculate the cumulated high-order corrections imposed by multipulse sequences adopted to increase the momentum separation of the interfering trajectories [2,3] or to enhance the instrument sensitivity at a specific frequency [26]. Second, we analyze the effect of more than quadratic external potentials in atom interferometers, a problem for which only a numerical solution has been proposed to date [27]. Small terms beyond uniform gravity are treated with perturbation theory, and the well-known case of the quadratic potential is used to validate our formulation. We demonstrate that the so-called "sensitivity function" in atom interferometry [28] gives the correct phase shift when the average over the initial velocity distribution is considered, even if it neglects a term of the Hamiltonian. We can also reinterpret the main phase shift terms in the commonly adopted path integral description of atom interferometry [13]. Evaluating the phase contribution of more than quadratic terms of the gravitational potential is relevant to several experiments where atoms are coherently manipulated close to the source masses [7,10,29-31].

The article is organized as follows. We describe our method based on the Heisenberg picture in Sec. II, where we consider the frequency chirp required to maintain the manipulation laser on resonance with the atoms and implement the unitary transformation that transfers the two interferometer trajectories on the classical mean path. Section III analyzes the wellknown case of atom interferometry in a quadratic potential and adopts another unitary transformation to separate the effects on the interferometric output due to the free evolution and to the pulses; the findings are compared with those reported in the literature. In Sec. IV we consider the effect of a more than quadratic external potential with a perturbative 
theory and generalize to arbitrary perturbative potentials the method proposed in Ref. [32] to mitigate the contrast loss due to the gravity gradient.

\section{UNITARY TRANSFORMATION: MEAN PATH}

In order to focus on the essential features of the calculation, we adopt a simplified two-level model in one dimension. Raman transitions between two stable levels $|1\rangle$ and $|2\rangle$ are characterized by a time-dependent Rabi frequency $\Omega(t)$, after adiabatic elimination of the excited level. The atoms are initially prepared in the internal state $|1\rangle$ and their initial wave function is assumed to be a Gaussian wave packet in momentum. The atoms have been prepared with an initial velocity selection pulse of length $\tau_{s}$, which fixes the momentum distribution width as $\sim m /\left(k \tau_{s}\right)$.

The Hamiltonian describing the effective two-level atom interacting with the Raman laser beams is [22]

$$
H=\left[\frac{\hat{p}^{2}}{2 m}+V(\hat{z})\right] I-\hbar \Omega(t) \cos \phi_{L}(\hat{z}, t) \sigma_{1}+\frac{\hbar \omega_{21}}{2} \sigma_{3},
$$

where $\hbar \omega_{21}$ is the energy difference between the two states, $\sigma_{i}$ are the Pauli matrices $(i=1,2,3)$, and $I$ is the identity matrix. We consider two cases for the external potential: $V(\hat{z})=m g \hat{z}-m \gamma \hat{z}^{2} / 2$, i.e., $V(\hat{z})$ at its most quadratic in $\hat{z}$, and $V(\hat{z})=m g \hat{z}+\mathcal{V}(\hat{z})$, where $\mathcal{V}(\hat{z})$ is sufficiently weak to be treated as a small perturbation. We assume that the laser fields are classical, so the noncommuting operators are only $\hat{z}$ and $\hat{p}$. To alleviate the notation, henceforth we drop the hat from $\hat{z}$ and $\hat{p}$ and their functions.

We consider a Kasevich-Chu-type interferometer [12], where a sequence of three pulses $\pi / 2-\pi-\pi / 2$ of temporal lengths $\tau, 2 \tau$, and $\tau$, respectively, are separated by two free evolution intervals of length $T-2 \tau$ so that the total duration of the interferometric sequence is $2 T$. We remark that different sequences of pulses can also be considered [16,17]. In present-day interferometers the orders of magnitude of $\tau$ and $T$ are $10^{-5}$ and $1 \mathrm{~s}$, respectively. We also assume $\tau_{s} \sim 10^{-4} \mathrm{~s}$.

In order to keep the optical field in resonance with the atoms during their free fall, a phase-continuous, linear frequency chirp on the laser fields partially compensates the Doppler effect. Thus, the phase $\phi_{L}$ can be written as a function of position and time as

$$
\phi_{L}(z, t)=\omega_{0} t+\frac{\alpha t^{2}}{2}-k z
$$

Here $\omega_{0}$ is the frequency difference between the two Raman beams, $k$ is the sum of the Raman beams wave numbers and $\alpha$ is the chirp rate. We make the simplifying assumption that $k$ is constant in time (which is the case if both Raman beams are frequency chirped in opposite directions), and we neglect any effect due to the finite speed of light. A recent discussion on this last point is found in Ref. [33].

Since in the following we frequently use unitary transformations, we recall that, under a generic unitary transformation $\left|\psi^{\prime}\right\rangle=U(t)|\psi\rangle$, the Hamiltonian transforms as

$$
H^{\prime}(t)=U(t) H U^{\dagger}(t)+i \hbar\left(\partial_{t} U\right) U^{\dagger}(t) .
$$

The time-evolution operator over the generic time interval $\left[t_{1}, t_{2}\right]$ obeys the differential equation

$$
i \hbar \partial_{t_{2}} \mathcal{U}\left(t_{2}, t_{1}\right)=H\left(t_{2}\right) \mathcal{U}\left(t_{2}, t_{1}\right),
$$

with the boundary condition $\mathcal{U}\left(t_{1}, t_{1}\right)=I$, whose general solution is the well-known time-ordered exponential [34]

$$
\mathcal{U}\left(t_{2}, t_{1}\right)=\mathcal{T} \exp \left(-\frac{i}{\hbar} \int_{t_{1}}^{t_{2}} H\left(t^{\prime}\right) d t^{\prime}\right) .
$$

Hereafter we distinguish the unitary time evolutions $\mathcal{U}\left(t_{2}, t_{1}\right)$ from the generic time-dependent unitary transformations denoted with $U_{n}(t)(n=1,2, \ldots)$. While $\mathcal{U}\left(t_{2}, t_{1}\right)$ is usually calculated through the Dyson series [34], we use the alternative Magnus expansion [35,36], for which $\mathcal{U}\left(t_{2}, t_{1}\right)$ is written as the exponential of a series:

$$
\mathcal{U}\left(t_{2}, t_{1}\right)=\exp \left(\sum_{n=1}^{+\infty} M_{n}\left(t_{2}, t_{1}\right)\right) .
$$

Differently from the Dyson series, the Magnus expansion preserves the unitarity of $\mathcal{U}\left(t_{2}, t_{1}\right)$ at any order, but, as a drawback, it requires an operator exponentiation. A summary on the Magnus expansion is found in Appendix A.

Under the generic time-dependent unitary transformation described above, the evolution operator is also transformed:

$$
\mathcal{U}^{\prime}\left(t_{2}, t_{1}\right)=U\left(t_{2}\right) \mathcal{U}\left(t_{2}, t_{1}\right) U^{\dagger}\left(t_{1}\right) .
$$

Following Ref. [22], the time-dependent phase $\phi_{L}(z, t)$ is eliminated by means of the unitary transformation generated by $U_{3}(t)=\exp \left[i \sigma_{3} \phi_{L}(z, t) / 2\right]$ (the index 3 indicates that the exponent is proportional to $\left.\sigma_{3}\right)$. After adopting the rotatingwave approximation [37] to cancel the terms oscillating as $\exp \left[i 2 \phi_{L}(z, t)\right]$, the Hamiltonian transformed under $U_{3}(t)$ reads

$$
\begin{aligned}
H^{\mathrm{I}}(t) & =U_{3}(t) H U_{3}^{\dagger}(t)-\frac{\hbar \partial_{t} \phi_{L}(z, t)}{2} \sigma_{3} \\
& =-\frac{\hbar \Omega(t)}{2} \sigma_{1}-\frac{\hbar}{2} \delta(t) \sigma_{3}+\left(\frac{p^{2}}{2 m}+\frac{\hbar^{2} k^{2}}{8 m}+V(z)\right) I,
\end{aligned}
$$

where $\delta(t)$ is defined as the Doppler-shifted detuning,

$$
\delta(t)=\Delta(t)+\frac{p k}{m},
$$

with $\Delta(t) \equiv \omega(t)-\omega_{21}$ and $\omega(t) \equiv \omega_{0}+\alpha t$. Note that the transformed momentum is $U_{3}(t) p I U_{3}^{\dagger}(t)=p I-\hbar k \sigma_{3} / 2$, so the transformation adds $\hbar k / 2$ to, and subtracts $\hbar k / 2$ from, the momentum of states $|1\rangle$ and $|2\rangle$, respectively: this is equivalent to a translation of the classical upper and lower trajectories on the mean path, i.e., the trajectory with average momentum after the first beam-splitter pulse, as shown in Fig. 1.

An additional unitary transformation will eliminate the term proportional to $I$ in $H^{\mathrm{I}}(t)$, which is equivalent to moving to a reference frame in free fall. This operation is straightforward if $V(z)$ is at its most quadratic in $z$; otherwise we must apply perturbation theory and assume that the potential is the sum of a large linear part and a small term. We consider the two cases separately. 


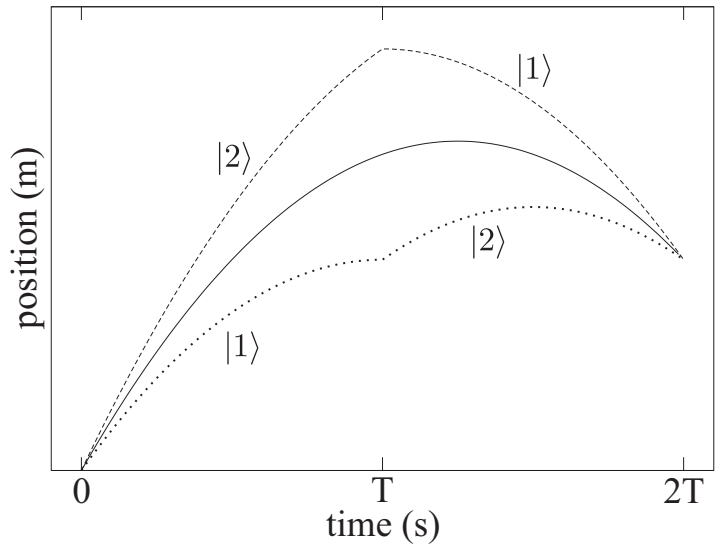

FIG. 1. Classical trajectories in a Kasevich-Chu [12] interferometer. The dashed and dotted lines represent the upper and lower interfering paths while the continuous line is the mean path, i.e., the trajectory of a particle with initial average momentum. At $t=T$ the $\pi$ pulse exchanges the internal states and the momentum with respect to the mean path. For a linear potential $V(z)$ the three trajectories converge to the same point at $t=2 T$; this condition is in general not valid for a nonlinear potential.

\section{QUADRATIC POTENTIAL}

We discuss the well-studied case of a quadratic potential to illustrate our method and derive with it well-known results.

For the earth's gravitational field we use the second-order potential $V(z)=m g z-m \gamma z^{2} / 2$, define $H_{\gamma}=\left(p^{2} / 2 m+\right.$ $\left.m g z-m \gamma z^{2} / 2\right) I$, and apply the unitary transformation $U_{0}(t)=\exp \left(i H_{\gamma} t / \hbar\right)$ to $H^{\mathrm{I}}$. Such transformation changes the reference system to the freely falling one, which is commonly adopted to describe the experiments in weightlessness [38,39]. The result is

$$
H^{\mathrm{II}}(t)=-\frac{\hbar}{2}\left[\Omega(t) \sigma_{1}+\delta(t) \sigma_{3}\right]
$$

where the momentum $p$ in $\delta(t)$ is now replaced by $p(t)$, i.e., the momentum time-evolved according to the Heisenberg representation with Hamiltonian $H_{\gamma}$ :

$$
p(t)=p \cosh \sqrt{\gamma} t+m z \sqrt{\gamma} \sinh \sqrt{\gamma} t-\frac{m g \sinh \sqrt{\gamma} t}{\sqrt{\gamma}} .
$$

As expected, this expression coincides at $t=0$ with the timeindependent $p$ operator. Similarly, for the following we define the time-evolved operator $z(t)$ :

$$
z(t)=z \cosh \sqrt{\gamma} t+p \frac{\sinh \sqrt{\gamma} t}{m \sqrt{\gamma}}+\frac{g(1-\cosh \sqrt{\gamma} t)}{\gamma} .
$$

In the case of the earth's gradient $\left(\gamma \simeq 3 \times 10^{-6} \mathrm{~s}^{-2}\right)$ and present-day interferometers $(T \simeq 1 \mathrm{~s})$, we have $2 T \sqrt{\gamma} \ll 1$; Eqs. (11) and (12) can then be expanded in series up to the second order in $\sqrt{\gamma} t$ and, keeping only terms at the most linear in $\gamma$, one obtains a simpler approximate expression for $\delta(t)$

$$
\delta(t) \simeq \Delta(0)+\frac{k p}{m}\left(1+\frac{\gamma t^{2}}{2}\right)-(g k-\alpha) t+k \gamma t\left(z-\frac{g t^{2}}{6}\right) .
$$

The expression above shows that, when $\alpha=g k$, the dominant time-dependent term in $\delta(t)$ is canceled and $\delta(t) \simeq \delta(0)$, which is equivalent to the atoms seeing a constant laser phase in their free fall. We remark that now $\left[\delta(t), \delta\left(t^{\prime}\right)\right] \equiv i c_{\delta, \delta}\left(t, t^{\prime}\right)$ is a c-number with $c_{\delta, \delta}\left(t, t^{\prime}\right)$ given by

$$
c_{\delta, \delta}\left(t, t^{\prime}\right)=k v_{r} \sqrt{\gamma} \sinh \sqrt{\gamma}\left(t-t^{\prime}\right) \simeq k v_{r} \gamma\left(t-t^{\prime}\right),
$$

where we have defined the recoil velocity $v_{r} \equiv \hbar k / m$.

We seek to separate the effect of the free evolution from that of the interferometer pulses. In this respect, the Hamiltonian of Eq. (10) is still unsatisfactory: while the term proportional to $\Omega(t)$ vanishes during the free evolution, its temporal integral, i.e., the corresponding accumulated phase, cannot be neglected since the pulses have an area of $\sim \pi$. Therefore, we define a third unitary transformation:

$$
\begin{gathered}
U_{1}(t) \equiv \exp \left[-i \phi_{1}(t) \sigma_{1} / 2\right], \\
\phi_{1}(t) \equiv \int_{0}^{t} \Omega\left(t^{\prime}\right) d t^{\prime},
\end{gathered}
$$

which leads to the Hamiltonian

$$
H^{\mathrm{III}}(t)=\frac{\hbar}{2} \delta(t)\left[\sin \phi_{1}(t) \sigma_{2}-\cos \phi_{1}(t) \sigma_{3}\right] .
$$

We see in the following that the Hamiltonian in Eq. (17) has the required form, i.e., the sum of a dominant term, $H_{L}^{\mathrm{III}}(t)$, proportional to $\sin \phi_{1}(t) \sigma_{2}$, plus a small term, $H_{S}^{\mathrm{III}}(t)$, proportional to $\cos \phi_{1}(t) \sigma_{3}$, which vanishes during the free evolution for pulses with ideal area. Later we refer to them as "ideal pulses."

\section{A. Approximate solution}

We aim to evaluate the transition probability for an atom in the initial internal state $|1\rangle$ to exit the interferometer in $|2\rangle$.

As a preliminary step, we neglect $H_{S}^{\mathrm{III}}(t)$ : as shown in Fig. 2, for ideal pulses $\cos \phi_{1}(t)=0$ during the free evolution, thus $H_{S}^{\mathrm{III}}(t)$ is a perturbation acting only during the interferometric pulses. We evaluate the corrections to this approximation in Sec. III B.

To evaluate the probability amplitude we need the offdiagonal matrix element of the evolution operator from $t=0$ to $t=2 T$, for which we revert to the Magnus expansion earlier introduced. As shown in Appendix A, since in the present approximation $\left[H(t), H\left(t^{\prime}\right)\right]$ is a c-number, the Magnus series terminates at $M_{2}$. Defining

$$
\begin{aligned}
& \phi_{2}(t) \equiv \int_{0}^{t} \delta\left(t^{\prime}\right) \sin \phi_{1}\left(t^{\prime}\right) d t^{\prime}, \\
& \psi_{2}(t) \equiv \frac{1}{8} \int_{0}^{t} d t^{\prime} \int_{0}^{t^{\prime}} c_{\delta, \delta}\left(t^{\prime}, t^{\prime \prime}\right) \sin \phi_{1}\left(t^{\prime}\right) \sin \phi_{1}\left(t^{\prime \prime}\right) d t^{\prime \prime},
\end{aligned}
$$




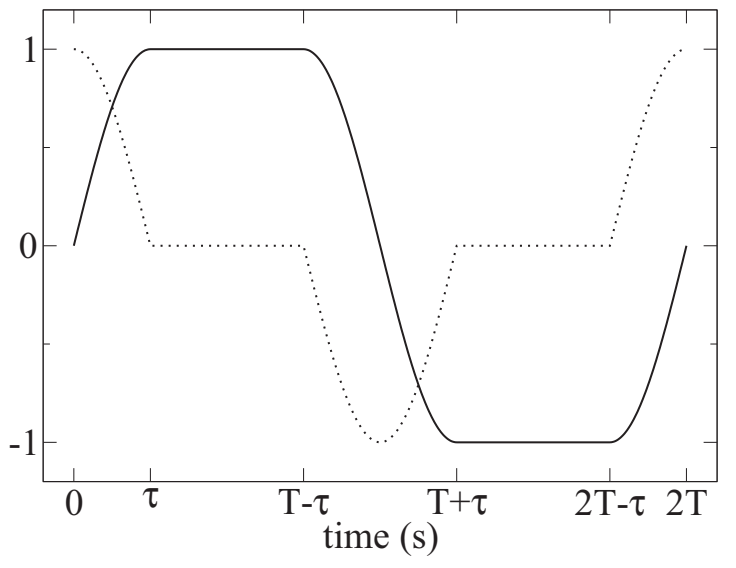

FIG. 2. Plot of the functions $\sin \phi_{1}(t)$ (continuous line) and $\cos \phi_{1}(t)$ (dotted line) for ideal rectangular pulses in a Mach-Zehnder interferometer. The two functions are formed either by sinusoidal functions or horizontal lines. In this figure $\eta=\tau / T$ is 0.25 for clarity; typical experimental values for $\eta$ are in the $10^{-4} \sim 10^{-5}$ range.

we have $M_{1}=-i \phi_{2} \sigma_{2} / 2$ and $M_{2}=-i \psi_{2} I$ (later, to simplify the notation, we omit the temporal arguments when $t=2 T$ ) and

$$
\mathcal{U}_{L}^{\mathrm{III}}(2 T, 0)=\exp \left(-i \psi_{2}\right) \exp \left(-i \frac{\phi_{2}}{2} \sigma_{2}\right),
$$

where the subscript $L$ means that we consider only $H_{L}^{\mathrm{III}}(t)$.

The evolution operator in the mean-path frame then reads $\mathcal{U}_{L}^{\mathrm{II}}(2 T, 0)=U_{1}^{\dagger}(2 T) \mathcal{U}_{L}^{\mathrm{III}}(2 T, 0)$. Since $U_{1}^{\dagger}(2 T)=$ $\exp \left[i \phi_{1} \sigma_{1} / 2\right]$, the transition probability $P_{21}$ from $|1\rangle$ to $|2\rangle$ at the output of the interferometric sequence can be evaluated directly:

$$
\begin{aligned}
P_{21} & =\left|\left\langle 2\left|\exp \left(i \phi_{1} \sigma_{1} / 2\right) \exp \left(-i \psi_{2}\right) \exp \left(-i \phi_{2} \sigma_{2} / 2\right)\right| 1\right\rangle\right|^{2} \\
& =\frac{1}{2}\left(1-\cos \phi_{1} \cos \phi_{2}\right),
\end{aligned}
$$

where the internal states $|n\rangle$, with $n=1$ or 2 , are evaluated in the reference frame II, i.e., $|n(t)\rangle=U_{0}(t) U_{3}(t)|n\rangle$.

Ideally, the total pulse area $\phi_{1}$ in Eq. (20) is equal to $2 \pi$ and the contrast $\cos \phi_{1}$ is equal to 1 . In the case of slightly imperfect pulses $\phi_{1}=2 \pi+\delta \phi_{1}$, the effect of $\delta \phi_{1}$ is just a contrast reduction of the interference fringes.

Assuming ideal, rectangular pulses, it is simple to obtain a closed-form expression for $\phi_{2}$ from Eqs. (9), (11), and (12). Here we report only an approximate expression using Eq. (13), keeping only terms up to the first order in the small parameter $\eta=\tau / T$. This expression depends only on the area, not on the actual shape, of the pulses:

$$
\begin{aligned}
\phi_{2}= & T^{2}\left(g k-\alpha-k \gamma z_{0}\right)\left(1-\frac{2 \pi-4}{\pi} \eta\right) \\
& -k \gamma T^{3}\left[v_{m}\left(1-\frac{2 \pi-4}{\pi} \eta\right)-g T\left(\frac{7}{12}-\frac{4 \pi-8}{3 \pi} \eta\right)\right],
\end{aligned}
$$

where we have used $p_{0} / m=v_{0}+v_{r} / 2=v_{m}$ for the motion on the mean path. We notice that some numerical coefficients in this formula do not agree with those in Eq. (40) of Ref. [25].
The explicit inclusion of $z$ shows that for a gradiometer where two clouds with the same initial velocity are separated by a distance $d$ the differential phase shift is simply

$$
\Delta \phi_{2}=-k \gamma d T^{2}\left(1-\frac{2 \pi-4}{\pi} \eta\right) .
$$

The formula for $P_{21}$ can be easily understood by noting that $H_{L}^{\mathrm{III}}(t)$ is diagonalized by the time-independent eigenvectors

$$
| \pm\rangle=\frac{|1\rangle \pm i|2\rangle}{\sqrt{2}} \Rightarrow|1\rangle=\frac{|+\rangle+|-\rangle}{\sqrt{2}},|2\rangle=\frac{|+\rangle-|-\rangle}{i \sqrt{2}},
$$

with the time-dependent eigenvalues

$$
E^{ \pm}(t)= \pm \frac{\hbar \delta(t) \sin \phi_{1}(t)}{2} .
$$

One must have, due to the interference between $|+\rangle$ and $|-\rangle$,

$$
P_{21}=\frac{1}{4}\left|1-\exp \left(\frac{i}{\hbar} \int_{0}^{2 T} E^{+}(t)-E^{-}(t) d t\right)\right|^{2},
$$

which is equivalent to Eq. (20) for ideal pulses. This is analogous to observing the Rabi oscillations in the dressed atom picture [40].

\section{B. Effect of the full Hamiltonian}

To take into account $H_{S}^{\mathrm{III}}(t)$ we apply another unitary transformation:

$$
U_{2}=\exp \left[i \psi_{2}(t)\right] \exp \left[i \phi_{2}(t) \sigma_{2} / 2\right] .
$$

Since $U_{2}^{\dagger}=\mathcal{U}_{L}^{\mathrm{III}}(t, 0)$, this unitary transformation is just the interaction representation with respect to $H_{L}^{\mathrm{III}}(t)$.

The new Hamiltonian $H^{\mathrm{IV}}(t)$ in the interaction representation is the transform of $H_{S}^{\mathrm{III}}(t)$,

$$
H^{\mathrm{IV}}(t)=-\frac{\hbar}{2} U_{2} \delta(t)\left[\cos \phi_{1}(t) \sigma_{3}\right] U_{2}^{\dagger},
$$

which can be evaluated using Eq. (A5), by letting $B=\delta$ and $A=\phi_{2} / 2$, as

$H^{\mathrm{IV}}(t)=\frac{\hbar}{4}\left[\left\{\delta(t), \sin \phi_{2}(t)\right\} \sigma_{1}-\left\{\delta(t), \cos \phi_{2}(t)\right\} \sigma_{3}\right] \cos \phi_{1}(t)$,

where $\{$,$\} denotes the anticommutator.$

Using the general identity to transform the evolution operators, we obtain

$\mathcal{U}^{\mathrm{IV}}(2 T, 0)=U_{2}(2 T) \mathcal{U}^{\mathrm{III}}(2 T, 0)=\left[\mathcal{U}_{L}^{\mathrm{III}}(2 T, 0)\right]^{\dagger} \mathcal{U}^{\mathrm{III}}(2 T, 0)$

or, equivalently,

$$
\mathcal{U}^{\mathrm{III}}(2 T, 0)=\mathcal{U}_{L}^{\mathrm{III}}(2 T, 0) \mathcal{U}^{\mathrm{IV}}(2 T, 0) .
$$

Therefore, $\mathcal{U}^{\mathrm{IV}}(2 T, 0)$ is the multiplicative correction sought to take into account $H_{S}^{\mathrm{III}}(t)$.

Under certain conditions, $\mathcal{U}^{\mathrm{IV}}(2 T, 0)$ is easily evaluated: if the pulses are ideal, during the free evolution we have 


$$
\begin{aligned}
& H^{\mathrm{IV}}(t)=0 \text { and } \mathcal{U}^{\mathrm{IV}}=I, \text { and thus, } \\
& \mathcal{U}^{\mathrm{IV}}(2 T, 0)=\mathcal{U}^{\mathrm{IV}}(2 T, 2 T-\tau) \mathcal{U}^{\mathrm{IV}}(T+\tau, T-\tau) \mathcal{U}^{\mathrm{IV}}(\tau, 0) .
\end{aligned}
$$

If the pulses are short, $\delta(t)$ can be considered constant during the pulses and we find

$$
\begin{aligned}
\mathcal{U}^{\mathrm{IV}}(2 T, 0)= & \exp \left\{-i \theta(2 T) \hat{n}\left[\phi_{2}(2 T)\right] \cdot \vec{\sigma}\right\} \\
& \times \exp \left\{2 i \theta(T) \hat{n}\left[\phi_{2}(T)\right] \cdot \vec{\sigma}\right\} \\
& \times \exp \left\{-i \theta(0) \hat{n}\left[\phi_{2}(0)\right] \cdot \vec{\sigma}\right\},
\end{aligned}
$$

where we have defined $\theta(t) \equiv \tau \delta(t) / 2$ and $\hat{n}\left[\phi_{2}(t)\right] \equiv$ [ $\left.\sin \phi_{2}(t), 0,-\cos \phi_{2}(t)\right]$. To alleviate the notation, we have written the half-anticommutators as products, e.g., $\theta(t) \hat{n}_{i}\left[\phi_{2}(t)\right]$ for $\left\{\theta(t), \hat{n}_{i}\left[\phi_{2}(t)\right]\right\} / 2$.

Clearly if $|\theta(t)| \ll 1$ then $\mathcal{U}^{\mathrm{IV}}(2 T, 0) \simeq I$. The effect of the correction $\mathcal{U}^{\mathrm{IV}}(2 T, 0)$ is to reduce the contrast in the interference fringes and to introduce an additional phase shift $\delta \phi_{2}$ with respect to Eq. (20). Such a phase shift can be evaluated explicitly by applying repeatedly the product rule for the exponential of Pauli vectors (see Appendix A) only if we assume $\gamma=0$ so all the commutators involving $\theta(t)$ and $\phi_{2}(t)$ are zero. Here we report only the approximate result when $|\theta(t)| \ll 1$ by expanding $P_{21}$ to leading-order terms in $\theta(t)$ and $\phi_{2}(\tau)$, which is of the same order as $\theta(\tau)$. After some algebra we obtain

$$
\delta \phi_{2}=-4 \theta^{2}(T) \sin 2 \phi_{2}(T)+O\left(\theta^{3}\right) .
$$

This is one of the main results of our analysis, showing that the interferometric phase shift carries an additional contribution due to the evolution during the laser pulses, actually dominated by the central $\pi$ pulse at time $t=T$. However, this contribution is easily washed out by averaging over the velocity distribution of the sample: under typical experimental conditions the width of the velocity distribution is inversely proportional to the duration $\tau_{s}$ of the selection pulse, and $\tau, \tau_{s}$, and $T$ obey to $\tau<\tau_{s} \ll T$; thus, we have simultaneously $|\theta(t)|^{2} \sim \tau^{2} / \tau_{s}^{2} \ll 1$ and $\phi_{2}(T) \sim$ $T / \tau_{s} \gg 1$, with $\phi_{2}(T)$ varying rapidly with the initial detuning $\delta(0)$. As a consequence, $\delta \phi_{2}$ averages to zero over the atomic sample and the phase shift evaluated in Eq. (21) still holds.

The effect of nonideal pulses has been considered in Ref. [41], for rectangular pulses, using the sensitivity function formalism, equivalent to our treatment in Sec. III A. There the terms proportional to $\delta(0)$ are retained and not assumed to cancel after the average over the initial velocity distribution.

\section{Loss of contrast}

In general, in a nonlinear potential, the end points of the upper and lower paths do not coincide. The loss of contrast induced by this effect and the strategies to mitigate it are discussed in Refs. [27,32] and experimentally implemented in Refs. $[42,43]$. Here we derive in our formalism the conditions to achieve high contrast in the case of a constant gradient, in order to extend them later to an arbitrary weak perturbing potential.

We start by evaluating the operators $z(t)$ and $p(t)$ after the unitary transformation generated by $H_{L}^{\mathrm{III}}$, using Eq. (A2) in
Appendix A, at time $t=2 T$ obtaining

$$
\begin{aligned}
z(2 T) & =z_{m}(2 T) I+\frac{i \sigma_{2}}{2}\left[\phi_{2}(2 T), z_{m}(2 T)\right] \\
& =z_{m}(2 T) I+\frac{\sigma_{2} v_{r}}{2} \int_{0}^{2 T} \sin \phi_{1}(t) \cosh \sqrt{\gamma}(2 T-t) d t \\
& \simeq z_{m}(2 T) I+\frac{\sigma_{2} v_{r} \gamma T^{3}}{2},
\end{aligned}
$$

and, similarly,

$$
p(2 T) \simeq p_{m}(2 T) I+\frac{\sigma_{2} m v_{r} \gamma T^{2}}{2} .
$$

The eigenvectors of both operators are again $| \pm\rangle$. The separation in position and momentum is given by the difference between the eigenvalues, i.e., $\Delta z(2 T)=v_{r} \gamma T^{3}$ and $\Delta p(2 T)=$ $m v_{r} \gamma T^{2}$.

In Ref. [27] it is shown that the conditions $\Delta z(2 T)=0$ and $\Delta p(2 T)=0$ at the end of an interferometric sequence ensure high contrast independently from the detection time. More generally, high contrast is obtained when $\Delta z(2 T)-$ $\Delta t_{d} \Delta p(2 T) / m=0$, where $\Delta t_{d}$ is the time interval between the last pulse and detection. By slightly changing the duration of the second free-evolution period it is possible to fulfill only the latter condition.

A better strategy, suggested in Ref. [32] and demonstrated in Refs. [42,43], is to change the momentum of the Raman beams by an amount $\delta k$ at the $\pi$ pulse. In this way $v_{r}$ is changed by an amount $\delta v_{r}=\hbar \delta k / m$ during the second free evolution: by choosing $\delta v_{r} / v_{r}=-\gamma T^{2} / 2, \Delta z(2 T)$ vanishes while the effect of $\Delta p(2 T)$ is negligible. Now, however, in Eq. (2) we have $k=k(t)$ and, due to the time derivative in Eq. (3), an extra term appears in the Hamiltonian, providing a momentum kick at the $\pi$ pulse that exactly compensates $\Delta p(2 T)$. The key to the possibility of compensating simultaneously $\Delta z(2 T)$ and $\Delta p(2 T)$ lies in the relation $m \Delta z(2 T) / \Delta p(2 T)=T$.

We show in Sec. IV C that this condition does not hold in general if $V(z)$ is more than quadratic.

\section{Comparison with previous results}

Here we show that Eq. (21) is consistent with previous literature.

Except for a sign, $\sin \phi_{1}(t)$ coincides with the sensitivity function introduced in Ref. [28] for rectangular pulses and it is immediately applicable to more general cases, i.e., Gaussian or imperfect pulses. Note that, even if the sensitivity function neglects $H_{S}^{\mathrm{III}}(t)$ in Eq. (19), the phase shift averaged over the initial atomic velocity distribution is correct as shown in Eq. (32).

If we use the expression for $\delta(t)$ given in Eq. (9) and, moving to the expectation values, apply the Ehrenfest's theorem replacing $p / m$ with $\dot{z}$, we can integrate by parts the first expression in Eq. (18) in the case of ideal rectangular pulses of negligible duration:

$$
\begin{aligned}
\phi_{2} & =-\int_{0}^{2 T}\left[\phi_{L}(t)+k z_{m}(t)\right] \Omega(t) \cos \phi_{1}(t) d t \\
& \simeq-D_{2}\left[\phi_{L}\right]-k D_{2}\left[z_{m}\right],
\end{aligned}
$$


where $\phi_{L}(t)$ is the primitive of $\Delta(t)$ and, to simplify the notation, we have defined $D_{2}[f] \equiv f(2 T)-2 f(T)+f(0)$. The boundary term of the integration by parts vanishes, for ideal pulses, as $\sin \phi_{1}(2 T)=\sin \phi_{1}(0)=0$. Note that in $D_{2}\left[\phi_{L}\right]$ the terms constant and linear in $t$ disappear so $-D_{2}\left[\phi_{L}\right]=\alpha T^{2}$, while, since $z(t)$ and $p(t)$ are linear in $z$ and $p$ in Eqs. $(11,12)$, then $2 z_{m}(t)=z_{u}(t)+z_{l}(t)$, so

$$
D_{2}\left[z_{m}\right]=\frac{z_{u}(2 T)+z_{l}(2 T)}{2}-\left[z_{u}(T)+z_{l}(T)\right]+z_{m}(0),
$$

which is the result given in Ref. [44].

Next, we compare Eq. (35) with the path integral prescription, as described, for example, in Ref. [15], where the phase shift is evaluated as the sum of three terms, $\delta \phi_{L}+\delta \phi_{p}+\delta \phi_{s}$. The "laser" term $\delta \phi_{L}$ is given by

$$
\begin{aligned}
\delta \phi_{L}= & \phi_{L}(0)+k z_{m}(0)-2 \phi_{L}(T)-k\left[z_{u}(T)+z_{l}(T)\right] \\
& +\phi_{L}(2 T)+k z_{l}(2 T),
\end{aligned}
$$

where $\phi_{L}(t) \equiv \phi_{L}(0, t)$ and $\phi_{L}(z, t)$ is given by Eq. (2).

The "propagation" term $\delta \phi_{p}$ is given by

$$
\delta \phi_{p}=\frac{1}{\hbar}\left(\int_{u} \mathcal{L} d t-\int_{d} \mathcal{L} d t\right) \equiv \frac{1}{\hbar} \oint_{\mathrm{cp}} \mathcal{L} d t
$$

where the two integrals are along the upper and lower classical paths and $\mathcal{L}$ is the Lagrangian. To simplify the notation the difference of the two integrals is denoted as a circulation integral along the classical path "cp", even if this can be open.

In the case of a quadratic potential it is easy to see that the kinetic and the potential energies give equal contributions to the integral so $\delta \phi_{p}=0$.

Finally the "separation" term is defined as

$$
\delta \phi_{s}=\frac{k\left[z_{u}(2 T)-z_{l}(2 T)\right]}{2},
$$

where we have taken into account that the average momentum of the two states in an output channel must be measured on the mean path. Clearly the path integral prescription gives the same result as Eq. (35).

Another possibility to evaluate $\phi_{2}$ involves integrating by parts the term $k p(t) / m$ in $\delta(t)$ in the other order, replacing $\dot{p}$ with $-\partial_{z} V$ and obtaining, in the same hypothesis as above, the contribution to $\phi_{2}$ due to $V, \phi_{2}^{V}$, as

$$
\phi_{2}^{V} \simeq \frac{v_{r}}{\hbar} \int_{0}^{2 T} S(t) \partial_{z} V(t) d t,
$$

where $S(t)$ is the primitive of $\sin \phi_{1}(t)$ (see Fig. 3). Noting that for a quadratic potential $V(z+\Delta z)-V(z-\Delta z)=$ $2 \Delta z \partial_{z} V(z)$ we can write

$$
\phi_{2}^{V}=\frac{1}{\hbar} \oint_{c} V(t) d t,
$$

where the closed path $c$ is delimited by $z_{m}(t) \pm v_{r} S(t) / 2$. We can also express $\phi_{2}^{V}$ as the difference of two integrals on the upper and lower classical paths by taking $z_{u}(t)-z_{l}(t) \equiv$

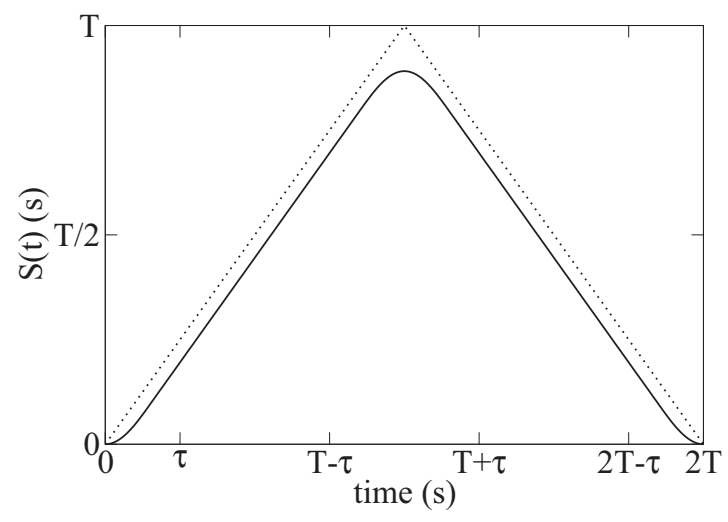

FIG. 3. Plot of the function $S(t)=\int_{0}^{t} \sin \phi_{1}\left(t^{\prime}\right) d t^{\prime}$ for $\eta=0.25$ (continuous line) and $\eta=0$ (dotted line) for square pulses.

$$
\begin{aligned}
& S(t) v_{r}+\delta z(t) \text { as a definition of } \delta z(t) \text { to obtain } \\
& \phi_{2}^{V}= \frac{1}{\hbar} \oint_{\mathrm{cp}} V(t) d t-\frac{1}{\hbar} \int_{0}^{2 T} \delta z(t) \partial_{z} V d t \\
&= \frac{1}{\hbar} \oint_{\mathrm{cp}} V(t) d t+\frac{p(2 T)\left[z_{u}(2 T)-z_{l}(2 T)\right]}{\hbar} \\
&-\frac{1}{\hbar} \int_{0}^{2 T} p(t) \delta v(t) d t,
\end{aligned}
$$

where $\delta v=\delta \dot{z}$. Note that $\delta v=0$ during the free evolution. Here the phase shift can be interpreted as a propagation term depending only on the potential, a separation term, and finally a term that contains the correction for the finite duration of the pulses.

\section{PERTURBATIVE POTENTIAL}

If the potential $V(z)=m g z+\mathcal{V}(z)$ is more than quadratic, a solution for the Heisenberg equations for $z$ and $p$ is in general not known, so it is not possible to transform to the free fall reference frame. Except for some special choice of $V(z)$, in general $\delta(t)$ will not be linear in both $z$ and $p$ so $\left[H(t), H\left(t^{\prime}\right)\right]$ will not be a c-number, preventing an exact calculation of $\mathcal{U}^{\mathrm{III}}$ as in Eq. (19).

Here we adopt a perturbative approach that works when $\mathcal{V}(z)$ is small, in a sense that is defined precisely later. In this way, we get an approximate result even for a purely quadratic potential, with the advantage of a much simpler algebra.

We use $U_{0}(t)$ generated by

$$
H_{m}=\left(\frac{p^{2}}{2 m}+m g z+\frac{\hbar^{2} k^{2}}{8 m}\right) I,
$$

obtaining

$$
H^{\mathrm{II}}(t)=-\frac{\hbar}{2}\left[\Omega(t) \sigma_{1}+\delta(t) \sigma_{3}\right]+\mathcal{V}(z) I,
$$

where in $H^{\mathrm{II}}(t)$ now $p(t)$ and $z(t)$ are given by Eqs. (11) and (12) when letting $\gamma \rightarrow 0$. This corresponds to a reference frame falling with constant acceleration $g$.

In the same way $\delta(t)$ is given by Eq. (13) with $\gamma=0$, so $c_{\delta, \delta}\left(t, t^{\prime}\right)=0$. 
The evaluation of $H^{\mathrm{III}}(t)$ is straightforward since $\left[U_{1}, \mathcal{V}(z) I\right]=0$ :

$$
H^{\mathrm{III}}(t)=\frac{\hbar}{2} \delta(t)\left[\sin \phi_{1}(t) \sigma_{2}-\cos \phi_{1}(t) \sigma_{3}\right]+\mathcal{V}(z) I .
$$

Finally we apply the last unitary transformation $U_{2}$ as outlined in Sec. III B. Note that, since now $\gamma=0$ all the commutators not involving $z(t)$ are zero, we can write

$$
H^{\mathrm{IV}}(t)=H_{\delta}^{\mathrm{IV}}(t)+H_{\mathcal{V}}^{\mathrm{IV}}(t),
$$

where $H_{\delta}^{\mathrm{IV}}(t)$ is given, as in the harmonic potential case, by Eq. (27), and

$$
H_{\mathcal{V}}^{\mathrm{IV}}(t)=\mathcal{V}^{+}\left(z, v_{r} S / 2\right) I+\mathcal{V}^{-}\left(z, v_{r} S / 2\right) \sigma_{2},
$$

where we have defined

$$
\mathcal{V}^{ \pm}(z, \Delta z) \equiv \frac{\mathcal{V}(z+\Delta z) \pm \mathcal{V}(z-\Delta z)}{2},
$$

and $H_{\mathcal{V}}^{\mathrm{IV}}(t)$ has been evaluated using Eqs. (A2) and (A8).

\section{A. Approximate solution}

Here we evaluate again the time-evolution operator $\mathcal{U}^{\mathrm{IV}}(2 T, 0)$ neglecting the $H_{S}^{\mathrm{IV}}(t)$ in $H_{\delta}^{\mathrm{IV}}(t)$. Later we take into account the full Hamiltonian.

We define $\mathcal{V}(z)$ as "small" when we can take $\left[\mathcal{V}^{ \pm}(t), \mathcal{V}^{ \pm}\left(t^{\prime}\right)\right]=0$ (see Appendix B) and truncate the Magnus series for $H_{\mathcal{V}}^{\mathrm{IV}}(t)$ to the first order: in this case the evolution operator is $\mathcal{U}^{\mathrm{IV}}(2 T, 0)=\exp \left[-i \epsilon_{0} I-i \epsilon_{2} \sigma_{2}\right]$, with

$$
\begin{aligned}
& \epsilon_{0}=\frac{1}{\hbar} \int_{0}^{2 T} \mathcal{V}^{+}\left(z, v_{r} S / 2\right) d t^{\prime}, \\
& \epsilon_{2}=\frac{1}{\hbar} \int_{0}^{2 T} \mathcal{V}^{-}\left(z, v_{r} S / 2\right) d t^{\prime} .
\end{aligned}
$$

To evaluate the transition probability $P_{12}$, we need to transform back to the previous reference frame, obtaining for the evolution operator

$$
\mathcal{U}^{\mathrm{III}}(2 T, 0)=\exp \left(-i \epsilon_{0}\right) \exp \left(-i \frac{\phi_{2}+2 \epsilon_{2}}{2} \sigma_{2}\right),
$$

where we have used the fact that, for ideal pulses, $\phi_{2}$ commutes with any analytic function of $z$ since $\left[z(t), \phi_{2}(2 T)\right]=$ $i v_{r} S(2 T)=0$, and that, since $\mathcal{V}$ is small, we can let $\left[\epsilon_{0}, \epsilon_{2}\right]=$ 0 and write $\mathcal{U}^{\mathrm{III}}(2 T, 0)=\mathcal{U}^{\mathrm{II}}(2 T, 0)$.

The term containing $\epsilon_{0}$ is an irrelevant phase factor, while $2 \epsilon_{2}$ is the additive phase shift to $\phi_{2}$ due to the perturbing potential $\mathcal{V}$. We can write $\epsilon_{2}$ using the Taylor series for $\mathcal{V}\left(z_{m}\right)$, i.e., on the mean path,

$$
2 \epsilon_{2}=\frac{2}{\hbar} \sum_{n=0}^{\infty} \frac{v_{r}^{2 n+1}}{(4 n+2) ! !} \int_{0}^{2 T} S^{2 n+1}(t) \partial_{z}^{2 n+1} \mathcal{V}\left(z_{m}\right) d t
$$

Note that the first term of the series above extends to $\mathcal{V}$ the results obtained for a quadratic potential while higher-order terms are present only when $\partial_{z}^{3} \mathcal{V} \neq 0$.

\section{B. Comparison with previous results}

The problem of nonquadratic potentials has been discussed in Ref. [27] locally solving for a quadratic potential but assuming time-varying values for $g$ and $\gamma$ along the atomic trajectories.

The density matrix approach in the Wigner representation has been adopted in various papers: by Dubetsky on the mean path $[17,45]$ and by others considering the evolution along the upper and lower paths $[27,46]$. The equivalence with the path integral approach has already been considered in Ref. [47] so we postpone a brief discussion on this subject until Appendix C.

\section{Loss of contrast}

To evaluate $z(2 T)$ after the unitary transformation $\mathcal{U}^{\mathrm{IV}}(2 T, 0)$, we note that $H_{\delta}^{\mathrm{IV}}$ does not contribute at $t=2 T$ for ideal pulses and the part proportional to $I$ in $H_{\mathcal{V}}^{\mathrm{IV}}$ has no effect on $\Delta z(2 T)$; we need then to evaluate only the commutator $\left[\epsilon_{2}, z(t)\right]$ with $\epsilon_{2}$ from Eq. (49).

From $\left[z(t), z\left(t^{\prime}\right)\right]=i \hbar\left(t^{\prime}-t\right) / m$, Eq. (A8) leads to

$$
\Delta z(2 T)=\frac{v_{r}}{m} \int_{0}^{2 T}(2 T-t) S(t) \partial_{z}^{2} \mathcal{V} d t,
$$

which generalizes the expression for $\Delta z(2 T)$ obtained in the quadratic case.

For $p(t)$, with the help of $\left[p(t), p\left(t^{\prime}\right)\right]=0$ and $\left[z(t), p\left(t^{\prime}\right)\right]=i \hbar$, we obtain

$$
\Delta p(2 T)=v_{r} \int_{0}^{2 T} S(t) \partial_{z}^{2} \mathcal{V} d t .
$$

In general it is not possible to have $m \Delta z(2 T) / \Delta p(2 T) \simeq$ $T$ if $\partial_{z}^{3} \mathcal{V} \neq 0$, so the scheme suggested in Ref. [32] is not extensible to arbitrary potentials but compensates only the average gradient over the classical trajectory. A straightforward modification, however, would be to use the change of $k$ in the $\pi$ pulse to cancel $\Delta z(2 T)$ and partially erase $\Delta p(2 T)$ and then change again $k$ at the last $\pi / 2$ pulse to complete the $\Delta p(2 T)$ compensation.

\section{Effect of the full Hamiltonian}

We can evaluate the effect of $H_{\delta}^{\mathrm{IV}}(t)$ by applying a unitary transformation that removes $H_{\mathcal{V}}^{\mathrm{IV}}(t)$ from Eq. (46) and, as in the quadratic case, obtain a resulting Hamiltonian $H^{V}$ which is nonzero only during the pulses. The evaluation of $H^{V}$ is straightforward if we make the approximation $\left[\mathcal{V}^{ \pm}(t), \delta\left(t^{\prime}\right)\right]=0$, justified in Appendix B. Analogously to the quadratic case, we obtain the new Hamiltonian

$$
H^{V}(t)=\frac{\hbar}{2} \delta(t) \cos \phi_{1}(t) \hat{n}\left[\phi_{2}(t)+2 \epsilon_{2}(t)\right] \cdot \vec{\sigma},
$$

which is the same as the one in Eq. (27) after the substitution $\phi_{2}(t) \rightarrow \phi_{2}(t)+2 \epsilon_{2}(t)$. Again we have assumed that all the operators are commuting so also Eqs. (31) and (32) and the relative considerations about sample averaging apply.

\section{CONCLUSIONS}

In a simple one-dimensional model we have addressed the effects of the finite duration of the interferometric pulses and of the presence of more than quadratic perturbative potentials in the calculation of the phase shift for atomic interferometers. 
In the case of quadratic potentials, we have recovered the already known interferometric phase shift, Eq. (21), for short pulses, i.e., to first order in $\tau / T$.

We have also shown that the finite duration of the pulses is accurately described by the sensitivity function method [28]: the additional phase shift generated by the part of the Hamiltonian it neglects, described by Eq. (32), vanishes when averaged over a typical initial velocity distribution of the atomic sample. Further, to take into account the finite pulse duration in the path integral formalism, we have derived Eq. (42).

We have also shown how our formalism naturally describes the final separation of the interferometer paths caused by the potential curvature and causing a contrast reduction in the interferometric fringes.

Finally, to lift the restriction of quadratic potentials we have evaluated perturbatively the phase shift due to an arbitrary weak potential, given by Eq. (51), that generalizes a similar result derived for quadratic potentials [13].

\section{ACKNOWLEDGMENTS}

We would like to thank B. Dubetsky for useful discussions, and B. Barrett, N. Gaaloul, and G. Lamporesi for a careful reading of the manuscript. A.B. acknowledges funding from Horizon 2020 QuantERA ERA-NET - Project TAIOL. M.P. acknowledges financial support from LAPHIA-IdEx Bordeaux.

\section{APPENDIX A: USEFUL FORMULAS}

Here we report for sake of completeness some useful formulas used in the article.

We start with the first three terms of the Magnus expansion:

$$
\begin{aligned}
M_{1}(t, 0)= & -\frac{i}{\hbar} \int_{0}^{t} H_{1} d t_{1}, \\
M_{2}(t, 0)= & -\frac{1}{2 \hbar^{2}} \int_{0}^{t} d t_{1} \int_{0}^{t_{1}}\left[H_{1}, H_{2}\right] d t_{2}, \\
M_{3}(t, 0)= & -\frac{i}{6 \hbar^{3}} \int_{0}^{t} d t_{1} \int_{0}^{t_{1}} d t_{2} \int_{0}^{t_{2}}\left[H_{1},\left[H_{2}, H_{3}\right]\right] \\
& +\left[H_{3},\left[H_{2}, H_{1}\right]\right] d t_{3},
\end{aligned}
$$

where $H_{n}$ is a shortened notation for $H\left(t_{n}\right)$. A recursion formula for generating successive terms is known [36].

Another identity that we often used is

$$
\exp (\alpha A) B \exp (-\alpha A)=\sum_{n=0}^{+\infty} \frac{\alpha^{n}}{n !} \operatorname{ad}_{A}^{n} B,
$$

where $\alpha$ is a complex number, $A$ and $B$ are operators and $\operatorname{ad}_{A}^{n} B$ is a nested commutator defined by recursion as

$$
\begin{aligned}
\operatorname{ad}_{A}^{n} B & =\left[A, \operatorname{ad}_{A}^{n-1} B\right], \quad n>0, \\
\operatorname{ad}_{A}^{0} B & =B .
\end{aligned}
$$

When $A$ and $B$ are Pauli matrices $\sigma_{i}$ and $\sigma_{j}$, respectively, with $i \neq j$ and $\alpha=i \theta$, it is easy to show that Eq. (A2) becomes

$$
\exp \left(i \theta \sigma_{i}\right) \sigma_{j} \exp \left(-i \theta \sigma_{i}\right)=\sigma_{j} \cos 2 \theta-\epsilon_{i j k} \sigma_{k} \sin 2 \theta .
$$

If $A$ and $B$ are scalar operators for which $[A,[A, B]]=0$, Eq. (A4) can be generalized to

$$
\begin{aligned}
& \exp \left(i A \sigma_{i}\right) B \sigma_{j} \exp \left(-i A \sigma_{i}\right) \\
& \quad=\frac{\{B, \cos 2 A\}}{2} \sigma_{j}-\frac{\{B, \sin 2 A\}}{2} \epsilon_{i j k} \sigma_{k} .
\end{aligned}
$$

We also remind that

$$
\exp (i \vec{n} \cdot \vec{\sigma})=I \cos n+i \vec{\sigma} \cdot \hat{n} \sin n,
$$

where $\vec{n}$ is a vector, $n$ its modulus, and $\hat{n}=\vec{n} / n$ the related unit vector. Note that, if $\vec{n}$ is a vector of operators, Eq. (A6) holds only if $\left[n_{i}, n_{j}\right]=0$.

The product of two of these matrices is

$$
\begin{aligned}
\exp (i \vec{n} \cdot \vec{\sigma}) \exp (i \vec{m} \cdot \vec{\sigma})= & I[\cos n \cos m-\hat{n} \cdot \hat{m} \sin n \sin m] \\
& +i[\hat{n} \sin n \cos m+\hat{m} \cos n \sin m \\
& -\hat{n} \times \hat{m} \sin n \sin m] \cdot \vec{\sigma}, \quad(\mathrm{A} 7)
\end{aligned}
$$

so it is of the same form as the two factors.

Another useful expression, if $[A, B]=c_{A B}$, where $c_{A B}$ is a c-number, and $f$ and $g$ are analytic functions, is [48]

$$
[f(A), g(B)]=-\sum_{n=1}^{\infty} \frac{\left(-c_{A B}\right)^{n}}{n !} \partial_{A}^{n} f(A) \partial_{B}^{n} g(B) .
$$

\section{APPENDIX B: PERTURBING POTENTIAL APPROXIMATIONS}

Here we discuss when the approximations involving the perturbing potential, namely, $\left[\mathcal{V}^{ \pm}(t), \mathcal{V}^{ \pm}\left(t^{\prime}\right)\right] \simeq 0$ and $\left[\mathcal{V}^{ \pm}(t), \delta\left(t^{\prime}\right)\right] \simeq 0$, assumed in Sec. IV, are justified. We need to show that the commutators above are negligible when compared with their anticommutators.

We start evaluating the following commutators when $\gamma=0$ :

$$
\begin{aligned}
{\left[z(t), z\left(t^{\prime}\right)\right] } & =\frac{\hbar}{m}\left(t-t^{\prime}\right), \\
{\left[z(t), \delta\left(t^{\prime}\right)\right] } & =i v_{r} .
\end{aligned}
$$

Note that the first commutator above defines a length scale $z_{0} \sim \sqrt{\hbar T / m}$ which, for heavy atoms, like Rb or Cs, and $T \sim$ $1 \mathrm{~s}$ is in the $25 \mu \mathrm{m}$ range.

Both $\mathcal{V}^{+}$and $\mathcal{V}^{-}$can be approximated with expressions evaluated on the mean path, $\mathcal{V}\left(z_{m}\right)$ and $S v_{r} \partial_{z} \mathcal{V}\left(z_{m}\right) / 2$, respectively. We can then apply Eqs. (A8) and (B1). Since $z_{0}$ is much smaller than the scale over which $\mathcal{V}$ is expected to vary significantly, we can keep only the first nonzero term in the sum in Eq. (A8), and note that, for example, $\left[\mathcal{V}^{+}(t), \mathcal{V}^{+}\left(t^{\prime}\right)\right]$ is of the order of $\delta \mathcal{V}^{+}(t) \delta \mathcal{V}^{+}\left(t^{\prime}\right)$ with $\delta \mathcal{V}^{+}$being the increment of $\mathcal{V}$ over a distance of the order of $z_{0}$. Almost everywhere on the mean path then $\mathcal{V}^{+}(t) \mathcal{V}^{+}\left(t^{\prime}\right) \gg \delta \mathcal{V}(t) \delta \mathcal{V}\left(t^{\prime}\right)$ holds. A similar argument can be applied to the other three combinations of signs in $\left[\mathcal{V}^{ \pm}(t), \mathcal{V}^{ \pm}\left(t^{\prime}\right)\right]$

For $\left[\mathcal{V}^{ \pm}(t), \delta\left(t^{\prime}\right)\right]$, choosing $A=z(t)$ and $B=\delta\left(t^{\prime}\right)$ in Eq. (A8), we need to show that $\left|\mathcal{V}^{ \pm}(t), \delta\left(t^{\prime}\right)\right| \gg\left|\partial_{z} \mathcal{V}^{ \pm}(t) v_{r}\right|$. Here we note that, in case of a sample of atoms that have been prepared with a velocity selection pulse of length $\tau_{s}$, as discussed in Sec. III B, on the average $\left|\delta\left(t^{\prime}\right)\right| \sim|\delta(0)| \sim 1 / \tau_{s}$, so we need again to compare $\mathcal{V}^{ \pm}(t)$ with $\delta \mathcal{V}^{ \pm}(t)$ where the 
increment is on a distance of the order $v_{r} \tau_{s}$. For our typical numbers such an increment is of the order of $1 \mu \mathrm{m}$ and, as above, the considerations on the smoothness of $\mathcal{V}^{ \pm}$over a short distance can be applied.

\section{APPENDIX C: EQUIVALENCE WITH THE WIGNER FUNCTION FORMALISM}

A review on the Wigner functions and quantum mechanics in phase space can be found in Ref. [49], and its specific application in atom interferometry can be found in Ref. [46]. Here we briefly summarize and compare previous results to ours. To avoid confusion, we restore hats to distinguish the operators from the variables of the Wigner function.

We point out that a convenient starting point for evaluating the Wigner function is not the initial Hamiltonian in Eq. (1) but rather Eq. (17), with the time-dependent operators $\hat{Z}=$ $\hat{z}(t)$ and $\hat{P}=\hat{p}(t)-m \Delta(t) / k$, with $\hat{z}(t)$ and $\hat{p}(t)$ defined in Eqs. (12) and (11), respectively. The two operators obey the canonical commutation relation $[\hat{Z}, \hat{P}]=i \hbar$, so we can use the Weyl transforms of $\hat{Z}$ and $\hat{P}$ as coordinates $z$ and $p$ in phase space. Moreover, since the transformation $\hat{z} \rightarrow \hat{Z}$ and $\hat{p} \rightarrow \hat{P}$ is linear, it is not only mapping the Heisenberg equation onto the Moyal equation but also acts as a coordinate change in phase space [50].

Here we show that neglecting $\hat{H}_{S}^{\mathrm{III}}$ in Eq. (17) leads readily to Eqs. (25) and (49) also in phase space.

To simplify the notation we introduce the spinorial Wigner functions associated with a generic initial density matrix, $\hat{\rho}(0)$ :

$$
W_{j k}(z, p ; 0) \equiv \frac{1}{2 \pi} \int e^{-i p u}\left\langle z-\frac{\hbar}{2} u\left|\hat{\rho}_{j k}(0)\right| z+\frac{\hbar}{2} u\right\rangle d u,
$$

where the indices $j$ and $k$ refer to the spinorial component in the basis $| \pm\rangle$ defined in Eq. (23).

In our case, at $t=0$ the spatial wave function is $\psi(z)$ and the spinorial state is $|1\rangle=(|+\rangle+|-\rangle) / \sqrt{2}$, thus the density matrix $\hat{\rho}(0)=|\psi ; 1\rangle\langle\psi ; 1|$ corresponds to a $2 \times 2$ Wigner function

$$
W_{j k}(z, p ; 0)=\frac{1}{2} f(z, p),
$$

with

$$
f(z, p)=\frac{1}{2 \pi} \int e^{-i p u} \psi^{*}\left(z-\frac{\hbar}{2} u\right) \psi\left(z+\frac{\hbar}{2} u\right) d u .
$$

Note that $f(z, p)$ is real. [49]

The temporal evolution of $W$ obeys the Moyal equation

$$
\partial_{t} W_{j k}=\frac{h_{j j} \star W_{j k}-W_{j k} \star h_{k k}}{i \hbar},
$$

[1] C. Bordé, Phys. Lett. A 140, 10 (1989).

[2] S.-W. Chiow, T. Kovachy, H.-C. Chien, and M. A. Kasevich, Phys. Rev. Lett. 107, 130403 (2011). where the $\star$-product is defined as

$$
a \star b=a \exp \left[\frac{i \hbar}{2}\left(\overleftarrow{\partial}_{z} \vec{\partial}_{p}-\overleftarrow{\partial}_{p} \vec{\partial}_{z}\right)\right] b
$$

with the arrows indicating if the derivative operators act on $a$ or $b$, and $h(t)=p(t) v_{r} \sin \phi_{1}(t) \sigma_{3} / 2$ is the Weyl transform of the Hamiltonian $\hat{H}=\hat{P} v_{r} \sin \phi_{1}(t) \sigma_{3} / 2$ in the $| \pm\rangle$ basis.

When $h$ is at its most quadratic in $z$ and $p$-actually just proportional to $p$ in our case-Eq. (C4) involving the diagonal elements of $W$ simplifies to the Liouville equation. The solutions for $W_{j j}$ are then $W_{j j}(z, p, t)=f\left(z-z_{j}^{c}, p-p^{c}\right)$, where $z_{j}^{c}(t)$ and $p^{c}(t)$ indicate the classical trajectories, namely,

$$
\begin{aligned}
& z_{ \pm}^{c}(t)= \pm \frac{v_{r} S(t)}{2} \\
& p^{c}(t)=p^{c}(0)=\frac{m \Delta(0)}{k} .
\end{aligned}
$$

For the off-diagonal elements we have

$$
W_{j k}(z, p ; t)=\exp \left[i \alpha_{k}(t)-i \alpha_{j}(t)\right] f(z, p),
$$

with

$$
\alpha_{ \pm}(t)=\frac{1}{\hbar} \int_{0}^{t} E^{ \pm}\left(t^{\prime}\right) d t^{\prime}
$$

and $E^{ \pm}$from Eq. (24).

With $W(z, p ; 2 T)$ we can calculate the transition probability

$$
\begin{aligned}
P_{21} & =\operatorname{Tr}[\hat{\rho}(2 T)|2\rangle\langle 2|] \\
& =\frac{1}{2} \int \operatorname{Tr}\left[W(z, p ; 2 T)\left(\begin{array}{rr}
1 & -1 \\
-1 & 1
\end{array}\right)\right] d z d p \\
& =\frac{1}{2} \int\left[W_{++}+W_{--}-2 \operatorname{Re}\left(W_{+-}\right)\right] d z d p \\
& =\frac{1-\cos \Delta \alpha(2 T)}{2},
\end{aligned}
$$

with $\Delta \alpha=\alpha_{+}-\alpha_{-}$, which is the same as Eq. (25).

When the weak potential $\mathcal{V}$ is added to $h(t)$ in Eq. (45), the correction $\Delta^{(1)} \alpha(t)$ in Eq. (C8) is obtained by applying perturbation theory at the first order as outlined in Refs. [49,51]:

$$
\begin{aligned}
\Delta^{(1)} \alpha(t) & =\frac{1}{\hbar} \int \mathcal{V}(z)\left[W_{++}(z, p, t)-W_{--}(z, p, t)\right] d z d p \\
& \simeq \frac{\mathcal{V}^{-}\left(z, z_{+}^{c}\right)}{\hbar},
\end{aligned}
$$

with $\mathcal{V}^{-}(z, \Delta z)$ defined as in Eq. (48), where we assumed that $W_{j j}$ is localized around $z_{j}^{c}$ and acts as $\delta\left(z-z_{j}^{c}\right)$ in the integral. The result is a correction term to $P_{21}$ which is the same as $2 \epsilon_{2}$ in Eq. (49).

The corrections to the classical trajectories $z_{ \pm}^{c}(t)$ due to $\mathcal{V}$ can instead be neglected at the first order in $\mathcal{V}$ according to the general rules of variational calculus, as discussed in Ref. [52].
[3] B. Plotkin-Swing, D. Gochnauer, K. E. McAlpine, E. S. Cooper, A. O. Jamison, and S. Gupta, Phys. Rev. Lett. 121, 133201 (2018). 
[4] T. Kovachy, P. Asenbaum, C. Overstreet, C. A. Donnelly, S. M. Dickerson, A. Sugarbaker, J. M. Hogan, and M. A. Kasevich, Nature (London) 528, 530 (2015).

[5] S. Dimopoulos, P. W. Graham, J. M. Hogan, and M. A. Kasevich, Phys. Rev. Lett. 98, 111102 (2007).

[6] B. Canuel et al., Sci. Rep. 8, 14064 (2018).

[7] G. Rosi, F. Sorrentino, L. Cacciapuoti, M. Prevedelli, and G. M. Tino, Nature (London) 510, 518 (2014).

[8] R. H. Parker, C. Yu, W. Zhong, B. Estey, and H. Müller, Science 360, 191 (2018).

[9] P. Hamilton, M. Jaffe, P. Haslinger, Q. Simmons, H. Muller, and J. Khoury, Science 349, 849 (2015).

[10] M. Jaffe, P. Haslinger, V. Xu, P. Hamilton, A. Upadhye, B. Elder, J. Khoury, and H. Müller, Nat. Phys. 13, 938 (2017).

[11] P. Cheiney, L. Fouché, S. Templier, F. Napolitano, B. Battelier, P. Bouyer, and B. Barrett, Phys. Rev. Appl. 10, 034030 (2018).

[12] M. A. Kasevich and S. Chu, Phys. Rev. Lett. 67, 181 (1991).

[13] P. Storey and C. Cohen-Tannoudji, J. Phys. II 4, 1999 (1994).

[14] C. Antoine and C. Bordé, Phys. Lett. A 306, 277 (2003).

[15] K. Bongs, R. Launay, and M. A. Kasevich, Appl. Phys. B 84, 599 (2006)

[16] M. Cadoret, N. Zahzam, Y. Bidel, C. Diboune, A. Bonnin, F. Théron, and A. Bresson, J. Opt. Soc. Am. B 33, 1777 (2016).

[17] B. Dubetsky and M. A. Kasevich, Phys. Rev. A 74, 023615 (2006).

[18] B. Cheng, P. Gillot, S. Merlet, and F. Pereira Dos Santos, Phys. Rev. A 92, 063617 (2015).

[19] V. Schkolnik, B. L. M. Hauth, C. Freier, and A. Peters, Appl. Phys. B 120, 311 (2015).

[20] C. J. Bordé, Gen. Relativ. Gravitation 36, 475 (2004).

[21] S. Dimopoulos, P. W. Graham, J. M. Hogan, and M. A. Kasevich, Phys. Rev. D 78, 042003 (2008).

[22] K.-P. Marzlin and J. Audretsch, Phys. Rev. A 53, 1004 (1996).

[23] A. Peters, High precision gravity measurements using atom interferometry, Ph.D. thesis, 1998.

[24] C. Antoine, Appl. Phys. B 84, 585 (2006).

[25] X. Li, C.-G. Shao, and Z.-K. Hu, J. Opt. Soc. Am. B 32, 248 (2015).

[26] P. W. Graham, J. M. Hogan, M. A. Kasevich, and S. Rajendran, Phys. Rev. D 94, 104022 (2016).

[27] A. Roura, W. Zeller, and W. P. Schleich, New J. Phys. 16, 123012 (2014).

[28] P. Cheinet, B. Canuel, F. Pereira Dos Santos, A. Gauguet, F. Yver-Leduc, and A. Landragin, IEEE Trans. Instrum. Meas. 57, 1141 (2008).

[29] M. A. Hohensee, B. Estey, P. Hamilton, A. Zeilinger, and H. Müller, Phys. Rev. Lett. 108, 230404 (2012).
[30] P. Asenbaum, C. Overstreet, T. Kovachy, D. D. Brown, J. M. Hogan, and M. A. Kasevich, Phys. Rev. Lett. 118, 183602 (2017).

[31] P. Haslinger, M. Jaffe, V. Xu, O. Schwartz, M. Sonnleitner, M. Ritsch-Marte, H. Ritsch, and H. Müller, Nat. Phys. 14, 257 (2017).

[32] A. Roura, Phys. Rev. Lett. 118, 160401 (2017).

[33] Y.-J. Tan, C.-G. Shao, and Z.-K. Hu, Phys. Rev. A 94, 013612 (2016).

[34] J. J. Sakurai and J. J. Napolitano, Modern Quantum Mechanics, 2nd ed. (Addison-Wesley, San Francisco, 2011).

[35] S. Blanes, F. Casas, J. A. Oteo, and J. Ros, Phys. Rep. 470, 151 (2009).

[36] S. Blanes, F. Casas, J. A. Oteo, and J. Ros, Eur. J. Phys. 31, 907 (2010).

[37] D. F. Walls and G. J. Milburn, Quantum Optics (SpringerVerlag, Berlin, 2008).

[38] D. Becker et al., Nature (London) 562, 391 (2018).

[39] B. Barrett, L. Antoni-Micollier, L. Chichet, B. Battelier, T. Lévèque, A. Landragin, and P. Bouyer, Nat. Commun. 7, 13786 (2016).

[40] C. Cohen-Tannoudji, J. Dupont-Roc, and G. Grynberg, AtomPhoton Interactions (Wiley \& Sons, New York, 2008).

[41] A. Bonnin, N. Zahzam, Y. Bidel, and A. Bresson, Phys. Rev. A 92, 023626 (2015).

[42] G. D’Amico, G. Rosi, S. Zhan, L. Cacciapuoti, M. Fattori, and G. M. Tino, Phys. Rev. Lett. 119, 253201 (2017).

[43] C. Overstreet, P. Asenbaum, T. Kovachy, R. Notermans, J. M. Hogan, and M. A. Kasevich, Phys. Rev. Lett. 120, 183604 (2018).

[44] C. Antoine and C. Bordé, J. Opt. B: Quantum Semiclass. Opt. 5, S199 (2003).

[45] B. Dubetsky, S. Libby, and P. Berman, Atoms 4, 14 (2016).

[46] E. Giese, W. Zeller, S. Kleinert, M. Meister, V. Tamma, and A. Roura, in Proceedings of the International School of Physics “Enrico Fermi”: Atom Interferometry, Vol. 188, edited by G. M. Tino and M. A. Kasevich (IOS, Amsterdam, 2014).

[47] B. Dubetsky, arXiv:1701.07909.

[48] M. K. Transtrum and J.-F. S. Van Huele, J. Math. Phys. 46, 063510 (2005).

[49] L. T. Curtright, D. B. Fairlie, and C. K. Zachos, A Concise Treatise on Quantum Mechanics in Phase Space (World Scientific, Singapore, 2014).

[50] N. C. Dias and J. N. Prata, J. Math. Phys. 45, 887 (2004).

[51] T. Curtright, T. Uematsu, and C. Zachos, J. Math. Phys. 42, 2396 (2001).

[52] D. M. Greenberger, W. P. Schleich, and E. M. Rasel, Phys. Rev. A 86, 063622 (2012). 